\title{
EXISTENCE OF NONOSCILLATORY RELATIVELY BOUNDED SOLUTIONS OF SECOND ORDER NEUTRAL DIFFERENTIAL EQUATIONS
}

\author{
Hussain Alimohamad ${ }^{1}$ \\ Bashar Ahmed Jawad Sharba ${ }^{2}$ \\ ${ }^{1}$ Department of Mathematics, College of science for Women, University of Baghdad, Iraq \\ ${ }^{2}$ Department of Mathematics, Faculty of Computer Science and Mathematics, University of \\ Kufa, Iraq
}

Recived : 14\3\2019

Revised : $26 \backslash 3 \backslash 2019$

Accepted : $1 \backslash 4 \backslash 2019$

Available online : $25 / 4 / 2019$

\begin{abstract}
In this paper some sufficient conditions are obtained to insure the existence of positive solutions which is relatively bounded from one side for nonlinear neutral differential equations of second order. We used the Krasnoselskii's fixed point theorem and Lebesgue's dominated convergence theorem to obtain new sufficient conditions for the existence of a Nonoscillatory one side relatively bounded solutions. These conditions are more applicable than some known results in the references. Three examples included to illustrate the results obtained.
\end{abstract}

Keywords: Existence of nonoscillatory solutions, Banach space, Neutral differential equations.

Mathematics Subject Classification: Differential Equations.

AMS Subject Classification: 34K40, 34K13 


\section{INTRODUTION}

This paper is concerned with the existence of a positive relatively bounded solution of the neutral differential equations of the form

$$
\begin{aligned}
& \left(a(t)\left(x(t)-q(t) x(\tau(t))^{\prime}\right)^{\prime}\right. \\
& -p(t) f\left(t, x(t), x(\sigma(t)), x^{\prime}(t), x^{\prime}(\sigma(t))\right)=0 .
\end{aligned}
$$

with respect to equation (1.1), throughout we shall assume the following:

(i) $p, q \in C\left(\left[t_{0},+\infty\right), \mathbb{R}^{+}\right), a \in C\left(\left[t_{0},+\infty\right),(0, \infty)\right), t \geq$ $t_{0}>0, t_{0} \in \mathbb{R}$.

(ii) $\quad \tau, \sigma \in C\left(\left[t_{0},+\infty\right), \mathbb{R}\right), \quad \tau(t) \leq t$, $\sigma(t) \leq t, \lim _{n \rightarrow \infty} \tau(t)=\infty, \lim _{n \rightarrow \infty} \sigma(t)=\infty$

(ii) $f \in C\left(\left[t_{0}, \infty\right) \times R^{4}, R\right), f$ is nondecreasing function, and $x f\left(t, x(t), x(\sigma(t)), x^{\prime}(t), x^{\prime}(\sigma(t))\right)>0, x \neq 0$. By a solution of Eq.(1.1) we mean a function $x \in$ $C\left[\left(t_{1}-\rho\left(t_{1}\right), \infty\right), \mathbb{R}\right), \quad \rho\left(t_{1}\right)=\min \{\tau(t), \sigma(t)\}$, forsome $t_{1} \geq t_{0}$, such that $a(t)(x(t)-q(t) x(t-\tau(t)))$ is continuously differentiable on $\left[t_{1}, \infty\right)$ and such that $x(t)$ satisfy Eq.(1.1) for $t \geq t_{1}$. A solution $x(t)$ is said to be nonoscillatory if it is either eventually positive or eventually negative that is there exists $t_{*} \geq t_{0}$, such that either $x(t)>0$ or $x(t)<0$ for all $t \geq t_{*}$, otherwise is said oscillatory [10].

Recently there have been a lot of activities concerning the existence of nonoscillatory solutions for neutral differential equations. In 1999, S. Tanaka [12] study the first order differential equations:

$$
\frac{d}{d t}[x(t)+h(t) x(\tau(t))]+\sigma f(t, x(g(t)))=0
$$

and established some sufficient conditions to insure the existence of positive solution of previous equation. In 2002, Y. Zhou, B. Zhang [14], found some sufficient conditions for the existence of nonoscillatory solutions the following equation:

$$
\begin{gathered}
\frac{d^{\mathrm{n}}}{d t^{\mathrm{n}}}[x(t)+c x(t-\tau)]+(-1)^{\mathrm{n}+1}[P(t) x(t-\sigma) \\
-Q(t) x(t-\delta)] .
\end{gathered}
$$

In 2005, Y. Yu, H. Wang [13], studied the nonoscillatory solutions of a class of second-order nonlinear neutral delay differential equations with positive and negative coefficients of the form:

$$
\begin{gathered}
\left(r(t)\left(x(t)+P(t) x(t-\tau)^{\prime}\right)^{\prime}+Q_{1}(t) \mathrm{f}\left(x\left(t-\sigma_{1}\right)\right)\right. \\
-Q_{2}(t) \mathrm{g}\left(x\left(t-\sigma_{2}\right)\right)=0
\end{gathered}
$$

In 2009, B. Dorociakova and R. Olach [5] studied the first order delay differential equations:

$$
\mathrm{x}^{\prime}(t)+p(t) x(t)+q(t) x(\tau(t))=0 .
$$

In the same year I. Culkov, L. Hanutiakov, R. Olach [3] studied the second order nonlinearneutral differential equations

$$
\frac{d^{2}}{d t^{2}}[x(t)-a(t) x(t-\tau)]=p(t) f(x(t-\sigma)) .
$$

In $2011, \mathrm{R}$. Olach etc. al [4], studied the first order neutral differential equations:

$$
\frac{d}{d t}[x(t)-a(t) x(t-\tau)]=p(t) f(x(t-\sigma))
$$

In 2012, L. Lietc. al[9], studied the existence of a bounded nonoscillatory positive solution for the equation.

$$
\begin{gathered}
\frac{d}{d t}[x(t)+a(t) x(t-\tau)]+p(t) f(x(t-\alpha)) \\
+q(t) g(x(t-\beta))=0
\end{gathered}
$$

In 2013, T. Canadan [1],obtained sufficient conditions for first-order nonlinear neutral differential equations to have nonoscillatory solutions for different ranges of $p_{1}(t)$ and $p_{2}(t, \xi)$ of the forms:

$$
\begin{gathered}
{\left[\left[x(t)-p_{1}(t) x(t-\tau)\right]^{\gamma}\right]^{\prime}+Q_{1}(t) \mathrm{G}(x(t-\sigma))=0} \\
{\left[\left[x(t)-p_{1}(t) x(t-\tau)\right]^{\gamma}\right]^{\prime}+\int_{\mathrm{c}}^{\mathrm{d}} Q_{2}(t) \mathrm{G}(x(t-\xi)) \mathrm{d} \xi=0}
\end{gathered}
$$

and

$$
\begin{aligned}
& {\left[\left[x(t)+\int_{\mathrm{a}}^{\mathrm{b}} p_{2}(t, \xi) x(t-\xi) \mathrm{d} \xi\right]^{\gamma}\right]^{\prime}} \\
& \quad+\int_{\mathrm{c}}^{\mathrm{d}} Q_{2}(t) \mathrm{G}(x(t-\xi)) \mathrm{d} \xi=0 \\
& \quad \frac{d}{d x}\left[x(t)+P_{1}(t) x\left(t-\tau_{1}\right)+P_{2}(t) x\left(t+\tau_{2}\right)\right] \\
& +Q_{1}(t) g_{1}\left(x\left(t-\sigma_{1}\right)\right)-Q_{2}(t) g_{2}\left(x\left(t+\sigma_{2}\right)\right)=0
\end{aligned}
$$

In 2017, F. Kong [8], studied the Existence of nonoscillatory solutions of a kind of first-order neutral differential equation:

$$
\begin{aligned}
& \frac{d}{d x}\left[x(t)+P_{1}(t) x\left(t-\tau_{1}\right)+P_{2}(t) x\left(t+\tau_{2}\right)\right] \\
& +Q_{1}(t) g_{1}\left(x\left(t-\sigma_{1}\right)\right)-Q_{2}(t) g_{2}\left(x\left(t+\sigma_{2}\right)\right)-f(t)=0 .
\end{aligned}
$$

In 2018, B. Çına and M. Tamer Şenel[2], obtained some sufficient conditions for the existence of positive solutions of variable coefficient nonlinear second order neutral differential equation with distributed deviating arguments of the form:

$$
\begin{gathered}
\left(x(t)-\int_{\mathrm{a}_{1}}^{\mathrm{b}_{1}} P(t, \xi) x(t-\xi) \mathrm{d} \xi\right)^{\prime \prime}+\int_{\mathrm{a}_{2}}^{\mathrm{b}_{2}} f(t, x(\sigma(t, \xi)) \mathrm{d} \xi \\
=0
\end{gathered}
$$

In this paper we prove that the existence of solution of Eq.(1.1) is relatively bounded, and we show that the solution is bounded from one side by function from above and below by function and ratio function respectively. some sufficient conditions for this purpose are obtained.

Definition 1.1 A function $x(t)$ is said to be relatively bounded from below (above) if there is a function $y(t)$ and constant $k$ such that $y(t) \leq x(t) \leq k(k \leq x(t) \leq y(t))$.

The following fixed point theorem and Lebesgue's dominated convergence theorem will be used to prove the main results in the next section.

Lemma 1.2[7] (Krasnoselskii's Fixed Point Theorem).

Let $X$ be a Banach space, let $\Omega$ be a bounded closed convex subset of $X$, and let $S_{1}, S_{2}$ be maps of $\Omega$ into $X$ such that $S_{1} x+S_{2} y \in \Omega$ for every pair $x, y \in \Omega$. If $S_{1}$ is 
contractive and $S_{2}$ is completely continuous, then the equation $S_{1} x+S_{2} x=x$ has a solution in $\Omega$.

Theorem 1.3 [11] (The Lebesgue Dominated Convergence Theorem)

Let $\left\{f_{n}\right\}$ be a sequence of measurable functions on $\mathrm{E}$. Suppose there is a function $g$ that is integrable over $\mathrm{E}$ and dominate $\left\{f_{n}\right\}$ on $\mathrm{E}$ in the sense that If $\left|f_{n}(x)\right| \leq g(x)$ on $\mathrm{E}$ for all $n$. If $\left\{f_{n}\right\} \rightarrow\{f\}$ pointwise a.e. on $\mathrm{E}$, then $f$ is integrable over $\mathrm{E}$ and

$\lim _{n \rightarrow \infty} \int_{E} f_{n}=\int_{E} f$.whereE is afinite measurable set.

\section{EXISTENCE OF ONE SIDE RELATIVELY BOUNDED SOLUTIONS:}

In this section we will establish several sufficient conditions to insure the existence of a nonoscillatory solutions which are one side relatively bounded by functions and a ratio of positive functions on $\left[t_{1}, \infty\right)$ of Eq.(1.1), $t_{1} \geq t_{0}$. Without loss of generality we will discuss the existence of eventually positive solution and the existence of eventually negative solution can be discussed in similar way.

The following conditions will be used in the next results:

H1. $0<q(t) \leq c<1$

H2. $M_{1} \leq f(t,.) \leq M_{2}, M_{1}, M_{2} \neq 0$, are constants.

H3. $m_{1} x(t) \leq f(t,.) \leq m_{2} x(t), m_{1}, m_{2} \neq 0$, are constants

Theorem 2.1. Suppose that $\mathrm{H} 1, \mathrm{H} 3$ hold, and there exist bounded function $u \in C^{1}\left(\left[t_{0}, \infty\right),[0, \infty)\right)$, a constant $N^{*}>0$, and $\rho\left(t_{1}\right) \geq t_{0}$ such that

$$
\begin{gathered}
u(t) \leq \frac{q\left(t_{1}\right) u\left(\tau\left(t_{1}\right)\right)}{q(t)} \\
\frac{u(t)-q(t) u(\tau(t))}{m_{1} \min _{t \geq t_{1}}\{u(t)\}} \leq \int_{t}^{\infty} \int_{s}^{\infty} \frac{p(\xi)}{a(\xi)} d \xi d s \\
\quad \leq \frac{1}{m_{2}}(1-q(t))
\end{gathered}
$$

Then Eq.(1.1) has a nonoscillatory relatively bounded from below.

Proof. Let $C\left(\left[t_{0},+\infty\right), \mathbb{R}\right)$ be the set of all continuous bounded functions with the norm $\|x\|=\sup _{t \geq t_{0}}|x(t)|$. Then $C\left(\left[t_{0},+\infty\right), \mathbb{R}\right)$ is a Banach space. We define a closed, bounded, and convex subset $\Omega$ of $C\left(\left[t_{0},+\infty\right), \mathbb{R}\right)$ as follows:

$$
\begin{aligned}
\Omega=\{x=x(t) \in & C\left(\left[t_{0},+\infty\right), \mathbb{R}\right): u(t) \leq x(t) \leq N^{*}, \\
& N^{*}>0, \\
& \left.t \geq t_{0}\right\} .
\end{aligned}
$$

For simplicity let

$f(t, \mathbf{x}(\mathrm{t}))=f\left(t, x(t), x(\tau(t)), x^{\prime}(t), x^{\prime}(t-\sigma(t))\right)$.

Now we define two maps $S_{1}$ and $S_{2}: \Omega \rightarrow$

$C\left(\left[t_{0},+\infty\right), \mathbb{R}\right)$ as follows:

$$
\left(\mathrm{S}_{1} x\right)(t)=\left\{\begin{array}{lr}
q(t) x(\tau(t)), & t \geq t_{1}, \\
\left(\mathrm{~S}_{1} x\right)\left(t_{1}\right), & t_{0} \leq t \leq t_{1},
\end{array}\right.
$$

$$
\begin{aligned}
& \left(S_{2} x\right)(t)= \\
& =\left\{\begin{array}{ll}
\int_{t}^{\infty} \int_{s}^{\infty} \frac{p(\xi)}{a(\xi)} f(\xi, \mathbf{x}(\xi)) d \xi d s \quad, t \geq t_{1}, \\
u(t)-q\left(t_{1}\right) u\left(\tau\left(t_{1}\right)\right)
\end{array}, t_{0} \leq t \leq t_{1},\right.
\end{aligned}
$$

We will show that for any $x, y \in \Omega$ we have $S_{1} x+S_{2} y \in$ $\Omega$.

From condition (2.2) it follows that for $t \geq t_{1}$

$$
\int_{t}^{\infty} \int_{s}^{\infty} \frac{p(\xi)}{a(\xi)} d \xi d s<\infty .
$$

For every $x, y \in \Omega$ and $t \geq t_{1}$, with regard (2.2) we obtain

$$
\begin{aligned}
\left(S_{1} x\right)(t)+ & \left(S_{2} y\right)(t) \\
& =q(t) x(\tau(t))+\int_{t}^{\infty} \int_{s}^{\infty} \frac{p(\xi)}{a(\xi)} f(\xi, \mathbf{y}(\xi)) d \xi d s \\
& \leq q(t) N^{*}+m_{2} N^{*} \int_{t}^{\infty} \int_{s}^{\infty} \frac{p(\xi)}{a(\xi)} d \xi d s \\
& \leq N^{*}\left(q(t)+m_{2} N^{*} \int_{t}^{\infty} \int_{s}^{\infty} \frac{p(\xi)}{a(\xi)} d \xi d s\right) \leq N^{*} .
\end{aligned}
$$

For $t \in\left[t_{0}, t_{1}\right]$, we have

$$
\begin{aligned}
\left(S_{1} x\right)(t)+\left(S_{2} y\right)(t) & =\left(S_{1} x\right)\left(t_{1}\right)+u(t)-q\left(t_{1}\right) u\left(\tau\left(t_{1}\right)\right) \\
& \leq q\left(t_{1}\right) x\left(\tau\left(t_{1}\right)\right)+N^{*}\left(1-q\left(t_{1}\right)\right) . \\
& \leq q\left(t_{1}\right) N^{*}+N^{*}\left(1-q\left(t_{1}\right)\right)=N^{*} .
\end{aligned}
$$

Furthermore, for $t \geq t_{1}$, with regard (2.2) we obtain

$$
\begin{aligned}
\left(S_{1} x\right)( & (t)+\left(S_{2} y\right)(t)= \\
= & q(t) x(\tau(t))+\int_{t}^{\infty} \int_{s}^{\infty} \frac{p(\xi)}{a(\xi)} f(\xi, \mathbf{y}(\xi)) d \xi d s, \\
\geq & q(t) u(\tau(t))+m_{1} \int_{t}^{\infty} \int_{s}^{\infty} \frac{p(\xi)}{a(\xi)} f(\xi, \mathbf{y}(\xi)) d \xi d s, \\
\geq & q(t) u(\tau(t))+m_{1} \int_{t}^{\infty} \int_{s}^{\infty} \frac{p(\xi)}{a(\xi)} \mathrm{y}(\xi) d \xi d s, \\
\geq & q(t) u(\tau(t))+m_{1} \int_{t}^{\infty} \int_{s}^{\infty} \frac{p(\xi)}{a(\xi)} u(\xi) d \xi d s, \\
\geq & q(t) u(\tau(t))+m_{1} \min _{t \geq t_{1}}\{u(t)\} \int_{t}^{\infty} \int_{s}^{\infty} \frac{p(\xi)}{a(\xi)} d \xi d s \\
\geq & u(t) .
\end{aligned}
$$


Hussain .A/Bashar.A

Let $t \in\left[t_{0}, t_{1}\right]$, from Eq.(2.4), with regard to (2.1) we get

$$
\begin{aligned}
\left(\mathrm{S}_{1} x\right)(t)+\left(\mathrm{S}_{2} y\right) & (t)= \\
= & \left(\mathrm{S}_{1} x\right)\left(t_{1}\right)+u(t)-q\left(t_{1}\right) u\left(\tau\left(t_{1}\right)\right) \\
& =q\left(t_{1}\right) x\left(\tau\left(t_{1}\right)\right)+u(t)-q\left(t_{1}\right) u\left(\tau\left(t_{1}\right)\right), \\
& \geq q\left(t_{1}\right) u\left(\tau\left(t_{1}\right)\right)+u(t)-q\left(t_{1}\right) u\left(\tau\left(t_{1}\right)\right) \\
& =u(t) .
\end{aligned}
$$

Thus, we have proved that $S_{1} x+S_{2} y \in \Omega$, for any $x, y \in \Omega$.

We will show that $S_{1}$ is a contraction mapping on $\Omega$. For $x, y \in \Omega$ and $t \geq t_{1}$ we have

$$
\begin{aligned}
\left\|\mathrm{S}_{1} x-\mathrm{S}_{1} y\right\| & =\sup _{t \geq t_{1}}\left|\left(\mathrm{~S}_{1} x\right)(t)-\left(\mathrm{S}_{1} y\right)(t)\right| \\
& =\sup _{t \geq t_{1}}|q(t) x(\tau(t))-q(t) y(\tau(t))| \\
& \leq \sup _{t \geq t_{1}} q(t)|x(\tau(t))-y(\tau(t))| \\
& \leq c\|x-y\|
\end{aligned}
$$

Also for $t \in\left[t_{0}, t_{1}\right]$.

$$
\begin{aligned}
& \quad\left\|\mathrm{S}_{1} x-\mathrm{S}_{1} y\right\|=\sup _{t_{0} \leq t \leq t_{1}}\left|\left(\mathrm{~S}_{1} x\right)(t)-\left(\mathrm{S}_{1} y\right)(t)\right| \\
= & \left|\left(\mathrm{S}_{1} x\right)\left(t_{1}\right)-\left(\mathrm{S}_{1} y\right)\left(t_{1}\right)\right| \\
= & \left|q\left(t_{1}\right) x\left(\tau\left(t_{1}\right)\right)-q\left(t_{1}\right) y\left(\tau\left(t_{1}\right)\right)\right| \\
= & q\left(t_{1}\right)\left|x\left(\tau\left(t_{1}\right)\right)-y\left(\tau\left(t_{1}\right)\right)\right| \\
\leq & c \sup _{t_{0} \leq t \leq t_{1}}|x(\tau(t))-y(\tau(t))| \\
= & c\|x-y\|
\end{aligned}
$$

Hence

$$
\left\|\mathrm{S}_{1} x-\mathrm{S}_{1} y\right\| \leq c\|x-y\| .
$$

Thus $S_{1}$ is a contraction mapping on $\Omega$.

To show that $S_{2}$ is completely continuous. First we will show that $S_{2}$ is continuous. By (2.5) and $\mathrm{H} 2$ it follows:

$$
\begin{aligned}
\int_{t}^{\infty} \int_{s}^{\infty} \frac{p(\xi)}{a(\xi)} f(\mathrm{~s}, & \mathbf{x}(\xi)) d \xi d s \\
& \leq m_{2} N^{*} \int_{t}^{\infty} \int_{s}^{\infty} \frac{p(\xi)}{a(\xi)} d \xi d s<\infty .
\end{aligned}
$$

Let $x_{k}=x_{k}(t) \in \Omega$ be such that $x_{k}(t) \rightarrow x(t)$ as $k \rightarrow \infty$. Because of $\Omega$ is closed, $x=x(t) \in \Omega$. For $t \geq t_{1}$ we have

$$
\begin{aligned}
& \left\|\left(\mathrm{S}_{2} x_{k}\right)(t)-\left(\mathrm{S}_{2} x\right)(t)\right\|=\sup _{t \geq t_{1}}\left|\left(\mathrm{~S}_{2} x_{k}\right)(t)-\left(\mathrm{S}_{2} x\right)(t)\right| \\
& =\sup _{t \geq t_{1}}\left|\int_{t}^{\infty} \int_{s}^{\infty} \frac{p(\xi)}{a(\xi)}\left(f\left(\xi, \mathbf{x}_{k}(\xi)\right)-f(\xi, \mathbf{x}(\xi))\right) d \xi d s\right|
\end{aligned}
$$

$\leq \int_{t_{1}}^{\infty} \int_{s}^{\infty} \frac{p(\xi)}{a(\xi)}\left|f\left(s, \mathbf{x}_{k}(\xi)\right)-f(s, \mathbf{x}(\xi))\right| d \xi d s$.

Since

$$
\left|f\left(\mathrm{~s}, \mathbf{x}_{k}(\xi)\right)-f(\mathrm{~s}, \mathbf{x}(\xi))\right| \rightarrow 0 \text { as } k \rightarrow \infty,
$$

by applying the Lebesgue dominated convergence theorem, we obtain

$$
\lim _{k \rightarrow \infty}\left\|\left(S_{2} x_{k}\right)(t)-\left(S_{2} x\right)(t)\right\|=0
$$

This means that $S_{2}$ is continuous.

Now to prove $S_{2} \Omega$ is relatively compact, we have to show that $\left\{S_{2} x: x \in \Omega\right\}$ is uniformly bounded and equicontinuous on $\left[t_{0}, \infty\right]$, according to Arzelã-Ascoli theorem [6]. It is clear that from (2.3) we get $\left\{S_{2} x: x \in\right.$ $\Omega\}$ is uniformly bounded. To show $\left\{S_{2} x: x \in \Omega\right\}$ is equicontinuouson $\left[t_{0}, \infty\right)$. Let $x \in \Omega$ and any $\varepsilon>0$, with regard to (2.8), there exists $t_{*} \geq t_{1}$ large enough so that

$\int_{\mathrm{t}}^{\infty} \int_{s}^{\infty} \frac{p(\xi)}{a(\xi)} f(\xi, \mathbf{x}(\xi)) d \xi d s<\frac{\varepsilon}{2}, \mathrm{t} \geq t_{*}$

Then, for $x \in \Omega, T_{2}>T_{1} \geq t_{*}$, we have

$$
\begin{gathered}
\left\|\left(\mathrm{S}_{2} x_{k}\right)\left(T_{2}\right)-\left(\mathrm{S}_{2} x\right)\left(T_{1}\right)\right\|= \\
\quad=\sup _{T_{2}>T_{1} \geq t_{*}}\left|\left(\mathrm{~S}_{2} x_{k}\right)\left(T_{2}\right)-\left(\mathrm{S}_{2} x\right)\left(T_{1}\right)\right| \\
\quad \leq\left|\left(\mathrm{S}_{2} x_{k}\right)\left(T_{2}\right)\right|+\left|\left(\mathrm{S}_{2} x\right)\left(T_{1}\right)\right| \\
\leq \int_{T_{2}}^{\infty} \int_{s}^{\infty} \frac{p(\xi)}{a(\xi)} f\left(\xi, \mathbf{x}_{k}(\xi)\right) d \xi d s \\
+\int_{T_{1}}^{\infty} \int_{s}^{\infty} \frac{p(\xi)}{a(\xi)} f(\xi, \mathbf{x}(\xi)) d \xi d s \\
<\frac{\varepsilon}{2}+\frac{\varepsilon}{2}=\varepsilon .
\end{gathered}
$$

For $x \in \Omega$ and $t_{1} \leq T_{1}<T_{2} \leq t_{*}$, we get

$$
\begin{aligned}
\|\left(\mathrm{S}_{2} x\right)\left(T_{2}\right)- & \left(\mathrm{S}_{2} x\right)\left(T_{1}\right) \|= \\
= & \sup _{t_{1} \leq T_{1}<T_{2} \leq t_{*}}\left|\left(\mathrm{~S}_{2} x\right)\left(T_{2}\right)-\left(\mathrm{S}_{2} x\right)\left(T_{1}\right)\right| \\
\leq & \sup _{t_{1} \leq T_{1}<T_{2} \leq t_{*}} \mid \int_{T_{2}}^{\infty} \int_{s}^{\infty} \frac{p(\xi)}{a(\xi)} f(\xi, \mathbf{x}(\xi)) d \xi d s \\
& -\int_{T_{1}}^{\infty} \int_{s}^{\infty} \frac{p(\xi)}{a(\xi)} f(\xi, \mathbf{x}(\xi)) d \xi d s \mid \\
\leq & \int_{T_{1}}^{T_{2}} \int_{s}^{\infty} \frac{p(\xi)}{a(\xi)} f(\xi, \mathbf{x}(\xi)) d \xi d s \\
\leq & m_{2} \max _{T_{1} \leq t \leq T_{2}}\left\{\frac{p(t)}{a(t)}\right\}\left(T_{2}-T_{1}\right) .
\end{aligned}
$$

Thus there exists $\delta_{1}=\frac{\varepsilon}{m_{T_{1} \leq t \leq T_{2}}\left\{\frac{p(t)}{a(t)}\right\}}$, such that

$$
\left|\left(\mathrm{S}_{2} x\right)\left(T_{2}\right)-\left(\mathrm{S}_{2} x\right)\left(T_{1}\right)\right|<\varepsilon \text {, if } 0<T_{2}-T_{1}<\delta_{1}
$$


Finally, for any $x \in \Omega, t_{0} \leq T_{1}<T_{2} \leq t_{1}$, and for any $\varepsilon>0$

$\left|\left(\mathrm{S}_{2} x\right)\left(T_{2}\right)-\left(\mathrm{S}_{2} x\right)\left(T_{1}\right)\right|=0<\varepsilon$, if $0<T_{2}-T_{1}<\delta_{2}$.

Hence, $\mathrm{S}_{2} \Omega$ is relatively compact. By Lemma 1.2 then Eq.(1.1) has a nonoscillatory relatively bounded from below. The proof is complete.

The next theorem we will give another new sufficient conditions to prove that the Eq.(1.1) has a nonoscillatory relatively bounded from above by $v(t)$.

Theorem 2.2.Suppose that $\mathrm{H} 1, \mathrm{H} 3$ hold, and there exist bounded function $v \in C^{1}\left(\left[t_{0}, \infty\right),[0, \infty)\right), \rho\left(t_{1}\right) \geq t_{0}$ such that

$$
\begin{aligned}
\frac{1}{m_{1}} & {[1-q(t)] \leq \int_{t}^{\infty} \int_{s}^{\infty} \frac{p(\xi)}{a(\xi)} d \xi d s } \\
& \leq \frac{1}{m_{2} \max _{t \geq t_{1}}\{v(t)\}}[v(t)-q(t) v(\tau(t))], t \geq t_{1} .
\end{aligned}
$$

Then Eq.(1.1) has a nonoscillatory relatively bounded from above.

Proof. Let $C\left(\left[t_{0},+\infty\right), \mathbb{R}\right)$ be the set of all continuous bounded functions with the norm $\|x\|=\sup _{t \geq t_{0}}|x(t)|$. Then $C\left(\left[t_{0},+\infty\right), \mathbb{R}\right)$ is a Banach space. Let $\Omega$ be a closed bounded, and convex subset of $C\left(\left[t_{0},+\infty\right), \mathbb{R}\right)$ defined as

$$
\Omega=\left\{x=x(t) \in C\left(\left[t_{0},+\infty\right), \mathbb{R}\right): N_{*} \leq x(t) \leq v(t),\right.
$$

$$
\left.N_{*}>0, \quad t \geq t_{0}\right\} . \quad \text { (2.11) }
$$

and the two maps $S_{1}$ and $S_{2}: \Omega \rightarrow C\left(\left[t_{0},+\infty\right), \mathbb{R}\right)$ defined as

$$
\left(\mathrm{S}_{1} x\right)(t)=\left\{\begin{array}{lr}
q(t) x(\tau(t)), & t \geq t_{1}, \\
\left(\mathrm{~S}_{1} x\right)\left(t_{1}\right), & t_{0} \leq t \leq t_{1},
\end{array}\right.
$$

$\left(\mathrm{S}_{2} x\right)(t)=$

$$
=\left\{\begin{array}{c}
\int_{t}^{\infty} \int_{s}^{\infty} \frac{p(\xi)}{a(\xi)} f(\xi, \mathbf{x}(\xi)) d \xi d s \quad, t \geq t_{1}, \\
v(t)-\mathrm{q}\left(t_{1}\right) v\left(\tau\left(t_{1}\right)\right) \quad, t_{0} \leq t \leq t_{1},
\end{array}\right.
$$

We will show that for any $x, y \in \Omega$ we have $S_{1} x+S_{2} y \in$ $\Omega$.

For every $x, y \in \Omega$ and $t \geq t_{1}$, we obtain

$\left(S_{1} x\right)(t)+\left(S_{2} y\right)(t)=$

$$
\begin{aligned}
& =q(t) x(\tau(t))+\int_{t}^{\infty} \int_{s}^{\infty} \frac{p(\xi)}{a(\xi)} f(\xi, \mathbf{y}(\xi)) d \xi d s \\
& \leq q(t) v(\tau(t))+m_{2} \int_{t}^{\infty} \int_{s}^{\infty} \frac{p(\xi)}{a(\xi)} \mathrm{y}(\xi) d \xi d s \\
& \leq q(t) v(\tau(t))+m_{2} \int_{t}^{\infty} \int_{s}^{\infty} \frac{p(\xi)}{a(\xi)} v(\xi) d \xi d s \\
& \leq q(t) v(\tau(t))+m_{2} \max _{t \geq t_{1}}\{v(t)\} \int_{t}^{\infty} \int_{s}^{\infty} \frac{p(\xi)}{a(\xi)} d \xi d s \\
& \leq q(t) v(\tau(t))+(v(t)-q(t) v(\tau(t)))=v(t)
\end{aligned}
$$

Let $t \in\left[t_{0}, t_{1}\right]$, using (2.12) we get

$$
\begin{aligned}
\left(S_{1} x\right)(t)+\left(S_{2} y\right) & (t)=\left(S_{1} x\right)\left(t_{1}\right)+v(t)-\mathrm{q}\left(t_{1}\right) v\left(\tau\left(t_{1}\right)\right) \\
\leq & \mathrm{q}\left(t_{1}\right) x\left(\tau\left(t_{1}\right)\right)+v(t)-\mathrm{q}\left(t_{1}\right) v\left(\tau\left(t_{1}\right)\right) \\
& =v(t) .
\end{aligned}
$$

Furthermore, for $t \geq t_{1}$, we get

$$
\begin{aligned}
\left(S_{1} x\right)(t)+ & \left(S_{2} y\right)(t)= \\
& =q(t) x(\tau(t))+\int_{t}^{\infty} \int_{s}^{\infty} \frac{p(\xi)}{a(\xi)} f(\xi, \mathbf{y}(\xi)) d \xi d s \\
& \geq q(t) N_{*}+m_{1} \int_{t}^{\infty} \int_{s}^{\infty} \frac{p(\xi)}{a(\xi)} \mathrm{y}(\xi) d \xi d s \\
& \geq q(t) N_{*}+\frac{m_{1} N_{*}}{a(t)} \int_{t}^{\infty} \int_{s}^{\infty} \frac{p(\xi)}{a(\xi)} d \xi d s \\
& \geq q(t) N_{*}+N_{*}(1-q(t)) \geq N_{*} .
\end{aligned}
$$

Then for $t \in\left[t_{0}, t_{1}\right]$. From equation (2.11) and (2.12), we have

$$
\begin{gathered}
\left(S_{1} x\right)(t)+\left(S_{2} y\right)(t)=\left(S_{1} x\right)\left(t_{1}\right)+v(t)-\mathrm{q}\left(t_{1}\right) v\left(\tau\left(t_{1}\right)\right) \\
\geq \mathrm{q}\left(t_{1}\right) x\left(\tau\left(t_{1}\right)\right)+v(t)-\mathrm{q}\left(t_{1}\right) v\left(\tau\left(t_{1}\right)\right) \\
\geq \mathrm{q}\left(t_{1}\right) N_{*}+N_{*}-\mathrm{q}\left(t_{1}\right) N_{*}=N_{*} .
\end{gathered}
$$

Thus we have proved that $S_{1} x+S_{2} y \in \Omega$ for any $x, y \in \Omega$. We can treat the rest of the proof in similar way as in the proof of theorem (2.1). By Lemma 1.2 there is an $x_{0} \in \Omega$ such that $S_{1} x_{0}+S_{2} x_{0}=x_{0}$. We conclude that $x_{0}(t)$ is a positive solution of (2.2). The proof is complete.

In the next theorem we will give another new sufficient conditions to prove that the Eq.(1.1) has a nonoscillatory one side relatively bounded from below by ratio function $\frac{u(t)}{a(t)}$.

Theorem2.3. Suppose that $\mathrm{H} 1, \mathrm{H} 2$ hold, and there exist bounded function $u \in C^{1}\left(\left[t_{0}, \infty\right),[0, \infty)\right), \rho\left(t_{1}\right) \geq$ $t_{0}$ such that

$$
\begin{gathered}
\frac{u\left(t_{1}\right)}{a\left(t_{1}\right)} \geq \frac{u(t)}{a(t)}, \quad t_{0} \leq t \leq t_{1} . \\
\frac{a(\tau(t)) u(t)-q(t) a(t) u(\tau(t))}{M_{1} a(t) a(\tau(t))} \leq \int_{t}^{\infty} \int_{s}^{\infty} \frac{p(\xi)}{a(\xi)} d \xi d s \\
\leq \frac{1}{M_{2}}(1-q(t)), t \geq t_{1} .
\end{gathered}
$$

Then Eq.(1.1) has a nonoscillatory relatively bounded from below.

Proof. Let $C\left(\left[t_{0},+\infty\right), \mathbb{R}\right)$ be the set of all continuous bounded functions with the norm $\|x\|=\sup _{t \geq t_{0}}|x(t)|$. Then $C\left(\left[t_{0},+\infty\right), \mathbb{R}\right)$ is a Banach space. We define a closed, bounded, and convex subset $\Omega$ of $C\left(\left[t_{0},+\infty\right), \mathbb{R}\right)$ as follows:

$$
\begin{aligned}
\Omega=\{x=x(t) \in & C\left(\left[t_{0},+\infty\right), R\right): \frac{u(t)}{a(t)} \leq x(t) \leq N^{*}, N^{*} \\
& \left.>0, t \geq t_{0}\right\} .
\end{aligned}
$$

The two maps $S_{1}$ and $S_{2}: \Omega \rightarrow C\left(\left[t_{0},+\infty\right), \mathbb{R}\right)$ defined as

$$
\begin{aligned}
& \left(\mathrm{S}_{2} x\right)(t)= \\
& =\left\{\begin{array}{c}
\int_{t}^{\infty} \int_{s}^{\infty} \frac{p(\xi)}{a(\xi)} f(\xi, \mathbf{x}(\xi)) d \xi d s \quad, t \geq t_{1} \\
\left(\mathrm{~S}_{2} x\right)\left(t_{1}\right) \quad, t_{0} \leq t \leq t_{1}
\end{array}\right.
\end{aligned}
$$$$
\left(\mathrm{S}_{1} x\right)(t)= \begin{cases}q(t) x(\tau(t)) & , t \geq t_{1} \\ \left(\mathrm{~S}_{1} x\right)\left(t_{1}\right) & , t_{0} \leq t \leq t_{1}\end{cases}
$$

We will show that for any $x, y \in \Omega$ we have $S_{1} x+S_{2} y \in$ $\Omega$. 
Hussain .A/Bashar.A

For every $x, y \in \Omega$ and $t \geq t_{1}$, we obtain

$\left(\mathrm{S}_{1} x\right)(t)+\left(\mathrm{S}_{2} y\right)(t)=$

$$
\begin{aligned}
& =q(t) x(\tau(t))+\int_{t}^{\infty} \int_{s}^{\infty} \frac{p(\xi)}{a(\xi)} f(\xi, \mathbf{y}(\xi)) d \xi d s \\
& \leq q(t) \mathrm{N}^{*}+\frac{M_{2}}{a(t)} \int_{t}^{\infty} \int_{s}^{\infty} \frac{p(\xi)}{a(\xi)} \mathrm{y}(\xi) d \xi d s \\
& \leq q(t) \mathrm{N}^{*}+\frac{M_{2} \mathrm{~N}^{*}}{a(t)} \int_{t}^{\infty} \int_{s}^{\infty} \frac{p(\xi)}{a(\xi)} d \xi d s \\
& \leq q(t) \mathrm{N}^{*}+\left(\mathrm{N}^{*}-q(t) \mathrm{N}^{*}\right)=\mathrm{N}^{*} .
\end{aligned}
$$

For $t \in\left[t_{0}, t_{1}\right]$, we have

$$
\left(S_{1} x\right)(t)+\left(S_{2} y\right)(t)=\left(S_{1} x\right)\left(t_{1}\right)+\left(S_{2} y\right)\left(t_{1}\right) \leq N^{*} .
$$

Furthermore, for $t \geq t_{1}$, we get

$\left(S_{1} x\right)(t)+\left(S_{2} y\right)(t)=$

$$
\begin{aligned}
& =q(t) x(\tau(t))+\int_{t}^{\infty} \int_{s}^{\infty} \frac{p(\xi)}{a(\xi)} f(\xi, \mathbf{y}(\xi)) d \xi d s \\
& \geq \frac{q(t)}{a(\tau(t))} u(\tau(t))+M_{1} \int_{t}^{\infty} \int_{s}^{\infty} \frac{p(\xi)}{a(\xi)} d \xi d s \\
& \geq \frac{q(t)}{a(\tau(t))} u(\tau(t))+\frac{a(\tau(t)) u(t)-q(t) a(t) u(\tau(t))}{a(t) a(\tau(t))} \\
& =\frac{u(t)}{a(t)} .
\end{aligned}
$$

Let $t \in\left[t_{0}, t_{1}\right]$. Using (2.13), we get

$$
\begin{aligned}
\left(\mathrm{S}_{1} x\right)(t)+\left(\mathrm{S}_{2} y\right)(t) & =\left(\mathrm{S}_{1} x\right)\left(t_{1}\right)+\left(\mathrm{S}_{2} y\right)\left(t_{1}\right) \\
& \geq \frac{u\left(t_{1}\right)}{a\left(t_{1}\right)} \geq \frac{u(t)}{a(t)} .
\end{aligned}
$$

Thus we have proved that $\mathrm{S}_{1} x+\mathrm{S}_{2} y \in \Omega$ for any $x, y \in$ $\Omega$.We can treat the rest of the proof in similar way as in the proof of theorem (2.1). By Lemma 1.2 there is an $x_{0} \in \Omega$ such that $S_{1} x_{0}+S_{2} x_{0}=x_{0}$. We conclude that $x_{0}(t)$ is a positive solution of Eq.(1.1). The proof is complete.

In the next theorem we will give another new sufficient conditions to prove that the Eq.(1.1) has a nonoscillatory relatively bounded from below by ratio function $\frac{v(t)}{a(t)}$.

Theorem2.4.Suppose that $\mathrm{H} 1, \mathrm{H} 3$ hold, and there exist bounded function $v \in C^{1}\left(\left[t_{0}, \infty\right),[0, \infty)\right), \rho\left(t_{1}\right) \geq t_{0}$ such that

$$
\begin{aligned}
& \frac{1}{m_{1}}(1-q(t)) \leq \int_{t}^{\infty} \int_{s}^{\infty} \frac{p(\xi)}{a(\xi)} d \xi d s \\
& \quad \leq \frac{a(\tau(t)) v(t)-q(t) a(t) v(\tau(\mathrm{t}))}{m_{2} \max _{t \geq t_{1}}\{v(t)\} a(t) a(\tau(t))}, t \geq t_{1} .
\end{aligned}
$$

Then Eq.(1.1) has a nonoscillatory relatively bounded from above.

Proof. Let $C\left(\left[t_{0},+\infty\right), \mathbb{R}\right)$ be the set of all continuous bounded functions with the norm $\|x\|=\sup _{t \geq t_{0}}|x(t)|$. Then $C\left(\left[t_{0},+\infty\right), \mathbb{R}\right)$ is a Banach space. We define a closed, bounded, and convex subset $\Omega$ of $C\left(\left[t_{0},+\infty\right), \mathbb{R}\right)$ as follows:

$$
\begin{gathered}
\Omega=\left\{x=x(t) \in C\left(\left[t_{0},+\infty\right), R\right): N_{*} \leq x(t) \leq \frac{v(t)}{a(t)}, t\right. \\
\left.\geq t_{0}, N_{*}>0\right\} . \text { (2.18) }
\end{gathered}
$$

We now define two maps $S_{1}$ and $S_{2}: \Omega \rightarrow$ $C\left(\left[t_{0},+\infty\right), \mathbb{R}\right)$ as follows:

$$
\begin{aligned}
& \left(\mathrm{S}_{1} x\right)(t)=\left\{\begin{array}{l}
q(t) x(\tau(t)) \quad, t \geq t_{1} \\
\left(\mathrm{~S}_{1} x\right)\left(t_{1}\right),
\end{array}, t_{0} \leq t \leq t_{1}\right. \\
& \left(\mathrm{S}_{2} x\right)(t)=\left\{\begin{array}{l}
\int_{t}^{\infty} \int_{s}^{\infty} \frac{p(\xi)}{a(\xi)} f(\xi, \mathbf{x}(\xi)) d \xi d s, t \geq t_{1} \\
\frac{v(t)}{a(t)}-q\left(t_{1}\right) \frac{v\left(\tau\left(t_{1}\right)\right)}{a\left(\tau\left(t_{1}\right)\right)}, t_{0} \leq t \leq t_{1}
\end{array}\right.
\end{aligned}
$$

We will show that for any $x, y \in \Omega$ we have $S_{1} x+S_{2} y \in$ $\Omega$.

For every $x, y \in \Omega$ and $t \geq t_{1}$, we obtain

$\left(S_{1} x\right)(t)+\left(S_{2} y\right)(t)=$

$$
\begin{aligned}
& =q(t) x(\tau(t))+\int_{t}^{\infty} \int_{s}^{\infty} \frac{p(\xi)}{a(\xi)} f(\xi, \mathbf{y}(\xi)) d \xi d s \\
& \leq \frac{q(t)}{a(\tau(t))} v(\tau(t))+m_{2} \int_{t}^{\infty} \int_{s}^{\infty} \frac{p(\xi)}{a(\xi)} \mathrm{y}(\xi) d \xi d s \\
& \leq \frac{q(t)}{a(\tau(t))} v(\tau(t))+m_{2} \int_{t}^{\infty} \int_{s}^{\infty} \frac{p(\xi)}{a(\xi)} v(\xi) d \xi d s \\
& \leq \frac{q(t)}{a(\tau(t))} v(\tau(t))+m_{2} \max _{t \geq t_{1}}\{v(t)\} \int_{t}^{\infty} \int_{s}^{\infty} \frac{p(\xi)}{a(\xi)} d \xi d s \\
& \leq \frac{q(t)}{a(\tau(t))} v(\tau(t))+\frac{a(\tau(t)) v(t)-q(t) a(t) v(\tau(t))}{a(t) a(\tau((t))} \\
& =\frac{v(t)}{a(t)} .
\end{aligned}
$$

For $t \in\left[t_{0}, t_{1}\right]$, we have

$\left(\mathrm{S}_{1} x\right)(t)+\left(\mathrm{S}_{2} y\right)(t)$

$$
\begin{gathered}
=\left(\mathrm{S}_{1} x\right)\left(t_{1}\right)+\frac{v(t)}{a(t)}-q\left(t_{1}\right) \frac{v\left(\tau\left(t_{1}\right)\right)}{a\left(\tau\left(t_{1}\right)\right)} \\
\leq \mathrm{q}\left(t_{1}\right) x\left(\tau\left(t_{1}\right)\right)+\frac{v(t)}{a(t)}-q\left(t_{1}\right) \frac{v\left(\tau\left(t_{1}\right)\right)}{a\left(\tau\left(t_{1}\right)\right)} \\
\leq \mathrm{q}\left(t_{1}\right) \frac{v\left(\tau\left(t_{1}\right)\right)}{a\left(\tau\left(t_{1}\right)\right)}+\frac{v(t)}{a(t)}-q\left(t_{1}\right) \frac{v\left(\tau\left(t_{1}\right)\right)}{a\left(\tau\left(t_{1}\right)\right)}=\frac{v(t)}{a(t)} .
\end{gathered}
$$

Furthermore, for $t \geq t_{1}$, we get

$$
\begin{aligned}
& \left(S_{1} x\right)(t)+\left(S_{2} y\right)(t)= \\
= & q(t) x(\tau(t))+\int_{t}^{\infty} \int_{s}^{\infty} \frac{p(\xi)}{a(\xi)} f(\xi, \mathbf{y}(\xi)) d \xi d s \\
\geq & q(t) N_{*}+\frac{M_{1}}{a(t)} \int_{t}^{\infty} \int_{s}^{\infty} \frac{p(\xi)}{a(\xi)} d \xi d s \\
\geq & q(t) N_{*}+N_{*}(1-q(t))=N_{*} .
\end{aligned}
$$

Let $t \in\left[t_{0}, t_{1}\right]$. From Eqs. (3.21) and (3.22), we get $\left(\mathrm{S}_{1} x\right)(t)+\left(\mathrm{S}_{2} y\right)(t)=$

$$
\begin{aligned}
& =\left(\mathrm{S}_{1} x\right)\left(t_{1}\right)+\frac{v(t)}{a(t)}-q\left(t_{1}\right) \frac{v\left(t_{1}-\tau\left(t_{1}\right)\right)}{a\left(t_{1}-\tau\left(t_{1}\right)\right)} \\
& =q\left(t_{1}\right) x\left(\tau\left(t_{1}\right)\right)+\frac{v(t)}{a(t)}-q\left(t_{1}\right) \frac{v\left(\tau\left(t_{1}\right)\right)}{a\left(\tau\left(t_{1}\right)\right)}
\end{aligned}
$$




$$
\begin{aligned}
& \geq q\left(t_{1}\right) N_{*}+\frac{v(t)}{a(t)}-q\left(t_{1}\right) \frac{v\left(\tau\left(t_{1}\right)\right)}{a\left(\tau\left(t_{1}\right)\right)} \\
& \geq q\left(t_{1}\right) N_{*}+\frac{v(t)}{a(t)}-q\left(t_{1}\right) N_{*}=N_{*} .
\end{aligned}
$$

Thus we have proved that $\mathrm{S}_{1} x+\mathrm{S}_{2} y \in \Omega$ for any $x, y \in \Omega$. We can treat the rest of the proof in similar way as in the proof of theorem 2.1. By Lemma 1.2 there is an $x_{0} \in$ $\Omega$ such that $S_{1} x_{0}+S_{2} x_{0}=x_{0}$. We conclude that $x_{0}(t)$ is a positive solution of Eq.(1.1). The proof is complete.

Example2.5. Consider the following nonlinear Neutral differential equation

$$
\begin{gathered}
\frac{d}{d x}\left(\frac{d}{d x}(a(t)(x(t)-q(t) x(t-2)))\right. \\
-p(t)\left(\frac{2}{\mathrm{t}}+1\right)=0 .
\end{gathered}
$$

Where $a(t)=e^{-1.5 t}, p(t)=\frac{1}{8} e^{-2 t}$, and $q(t)=0.3$, Let $u(t)=e^{-0.5 t}, 1 \leq f(t, \mathbf{x}(\mathrm{t}))=\frac{2}{\mathrm{t}}+1 \leq 3, t \geq t_{1}=1$.

Solution: It is clear that condition (2.1) holds, since

$$
e^{-0.5 t} \leq e^{-0.5\left(t_{1}-2\right)}, t_{0} \leq t \leq t_{1}
$$

To show condition (2.2) oftheorem (2.1) verified:

$$
\begin{aligned}
\frac{1}{M_{1}}(u(t)-q(t) u(\tau(t))) & \leq \int_{t}^{\infty} \int_{s}^{\infty} \frac{p(\xi)}{a(\xi)} d \xi d s \\
& \leq \frac{1}{M_{2}}(1-q(t)), \quad t \geq 1,
\end{aligned}
$$

Let $R_{1}(\mathrm{t})=\frac{1}{M_{1}}(u(t)-q(t) u(\tau(t))), R_{2}(\mathrm{t})=0.5 e^{-0.5 t}$, and

$$
R_{3}(\mathrm{t})=\frac{1}{M_{2}}(1-q(t)) .
$$

Then $R_{1}(\mathrm{t}) \leq R_{2}(\mathrm{t}) \leq R_{3}(\mathrm{t})$, for $t \geq 1$ so all conditions of Theorem 2.1 hold, by Theorem there exists a positive solution of Eq.(2.20).

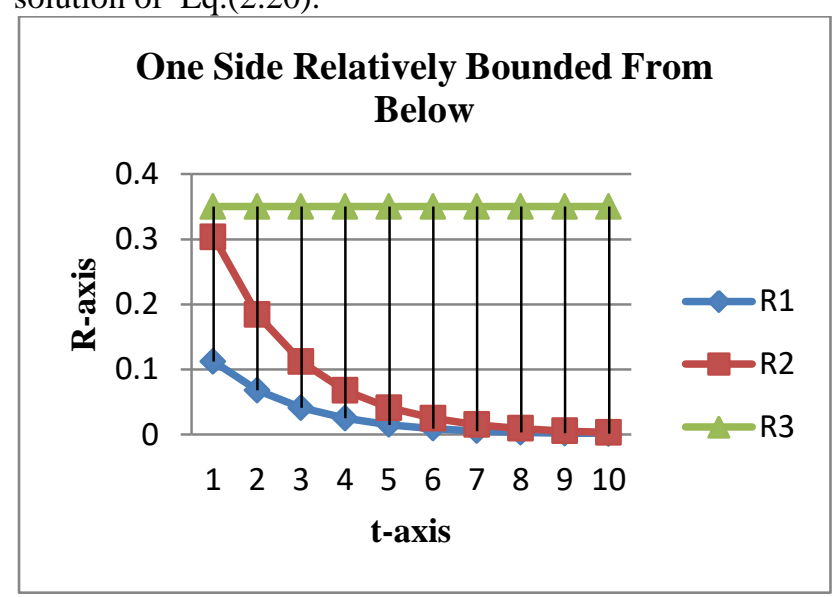

Figure 2.1: Thegraph of $R_{1}(\mathrm{t}), R_{2}(\mathrm{t})$, and $R_{3}(\mathrm{t})$, of theorem (2.1)

Example 2.6. Consider the following nonlinear Neutral differential equation

$\frac{d}{d t}\left(\frac{d}{d t}(a(t)(x(t)-q(t) x(t-1)))\right)-$

$$
p(t)\left(\frac{1}{2} \sin t+1.5\right)=0, \quad t \geq 0,
$$

where $a(t)=\frac{-(10+5 t)}{3}, \quad p(t)=\frac{2}{(2+t)^{2}}, \quad q(t)=0.5, \quad$ and $v(t)=2-e^{-t}, 1 \leq f(t, \mathbf{x}(\mathrm{t}))=\frac{1}{2} \sin t+1.5 \leq 2, t_{1}=$ 2.

Solution. To show condition (2.10) of theorem (2.2) verified:

$$
\begin{gathered}
\frac{1}{m_{1}}[1-q(t)] \leq \int_{t}^{\infty} \int_{s}^{\infty} \frac{p(\xi)}{a(\xi)} d \xi d s \\
\quad \leq \frac{[v(t)-q(t) v(\tau(t))]}{m_{2} \max _{t \geq t_{1}}\{v(t)\}}, t \geq t_{1} . \\
\text { Let } R_{1}(\mathrm{t})=\frac{1}{m_{1}}[1-q(t)], R_{2}(\mathrm{t})=\frac{1+0.8 t}{2+\mathrm{t}} \text { and } \\
R_{3}(\mathrm{t})=\frac{[v(t)-q(t) v(\tau(t))]}{m_{2} \max _{t \geq t_{1}}\{v(t)\}} .
\end{gathered}
$$

Then $R_{1}(\mathrm{t}) \leq R_{2}(\mathrm{t}) \leq R_{3}(\mathrm{t})$, for $t \geq 1$ so all conditions of Theorem 2.2 hold, by Theorem there exists a positive solution of Eq.(2.21).

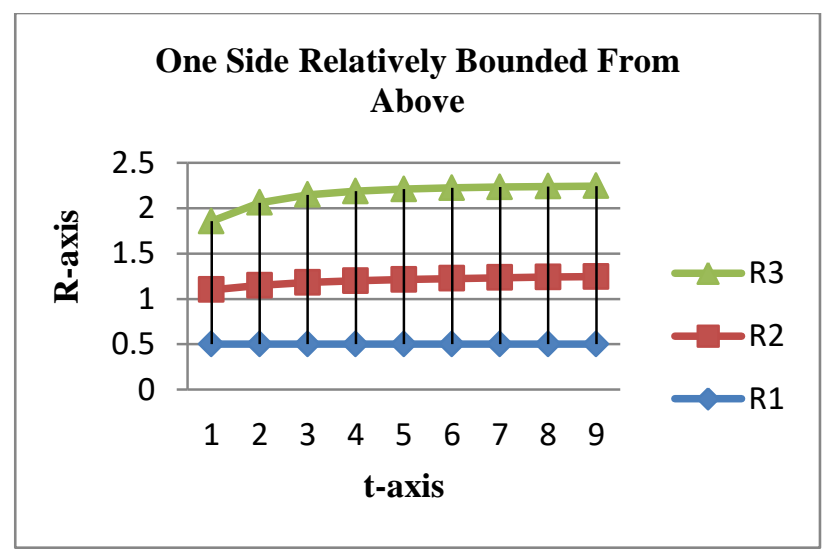

Figure 2.2: Thegraph of $R_{1}(\mathrm{t}), R_{2}(\mathrm{t})$, and $R_{3}(\mathrm{t})$, of theorem (2.2)

Example 2.7. Consider the following nonlinear Neutral differential equation

$$
\begin{aligned}
& \frac{d}{d t}\left(\frac{d}{d t}(a(t)(x(t)-q(t) x(t-1)))\right) \\
- & p(t)(\cos t+1)=0, \quad t \geq 0,
\end{aligned}
$$

Where $a(t)=2^{-1.5 t}, p(t)=2^{-3 t-3}$, and $q(t)=0.5$, and $u(t)=2^{-0.5 t}, 1 \leq f(t, \mathbf{x}(\mathrm{t}))=\cos t+1 \leq 2, t_{1}=2$.

Solution:It is clear thatcondition (2.13)holds, since

$$
\frac{u\left(t_{1}\right)}{a\left(t_{1}\right)}-\frac{u(t)}{a(t)}=\frac{2^{-0.5 t_{1}}}{2^{-1.5 t_{1}}}-\frac{2^{-0.5 t}}{2^{-1.5 t}} \geq 0,0 \leq t \leq 2 .
$$

To show condition (2.14) of theorem (2.3) verified:

$$
\begin{gathered}
\frac{a(\tau(t)) u(t)-q(t) a(t) u(\tau(t))}{M_{1} a(t) a(t-\tau)} \leq \int_{t}^{\infty} \int_{s}^{\infty} \frac{p(\xi)}{a(\xi)} d \xi d s \\
\leq \frac{1}{M_{2}}(1-q(t)), t \geq t_{1} . \\
\text { Let } R_{1}(\mathrm{t})=\frac{a(\tau(t)) u(t)-q(t) a(t) u(\tau(t))}{M_{1} a(t) a(\tau(t))},
\end{gathered}
$$


and

$$
R_{2}(\mathrm{t})=\frac{2^{-0.5 t-3}}{\ln (2)}
$$

$$
R_{3}(\mathrm{t})=\frac{1}{M_{2}}(1-q(t)) \text {. }
$$

Then $R_{1}(\mathrm{t}) \leq R_{2}(\mathrm{t}) \leq R_{3}(\mathrm{t})$, for $t \geq 1$ so all conditions of Theorem 2.3 hold, by Theorem there exists a positive solution of Eq.(2.22).

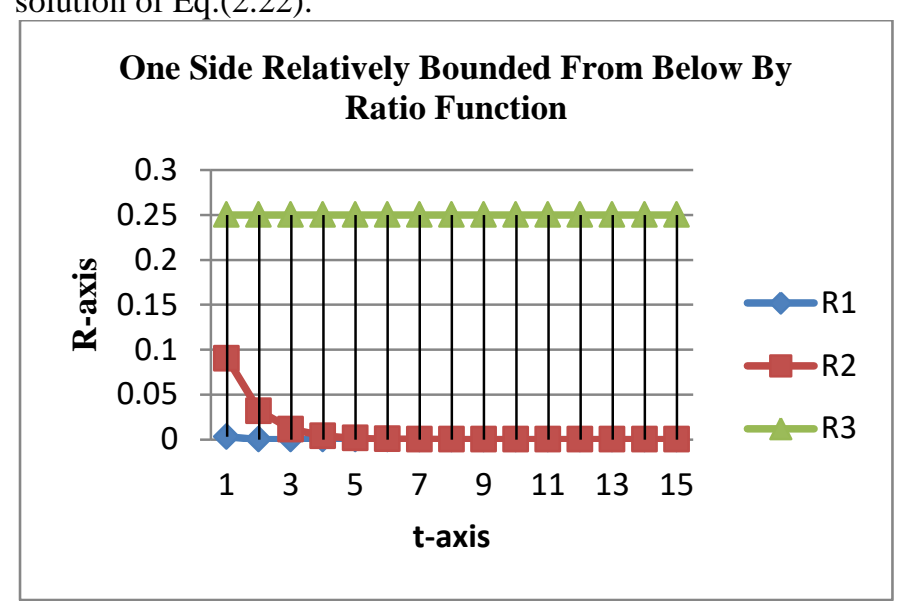

Figure 2.3: Thegraph of $R_{1}(\mathrm{t}), R_{2}(\mathrm{t})$, and $R_{3}(\mathrm{t})$, of theorem (2.3).

\section{REFERENCES}

1. T. Candan, Existence of Nonoscillatory Solutions of First-Order Nonlinear Neutral Differential Equations, Appl. Math. Lett., 26, 1182-1186, (2013).

2. Çina and M. Tamer Şenel, Positive Solutions of Second-order Neutral Differential Equations with Distributed Deviating Arguments, Journal of Institue

3. I. Culkov, L. Hanutiakov, R. Olach, Existence for Positive Solutions of Second Order Neutral Nonlinear Differential Equations, Applied Mathematics Letters, 22, 1007-1010, (2009).
4. Dorociakova B., A. Najmanova and R. Olach, Existence of Nonoscillatory Solutions of First Order Neutral Differential Equations, Abstract and Applied Analysis, 2011, 1-9,(2011).

5. B. Dorociakova and R. Olach, Existence of Positive Solutions of Delay Differential Equations, Tatra Mt. Math. Publ., 43, 63-70, (2009).

6. L. Erbe H., Q. Kong, and B. G. Zhang, Oscillation Theory for Functional Differential Equations, vol. 190 of Monographs and Textbooks in Pure and Applied Mathematics, Marcel Dekker, New York, NY, USA, (1995).

7. I. Gyori and G. Ladas, Oscillation Theory of Delay Differential Equations, Clarendon Press, Oxford , (1991).

8. F. Kong, Existence of non-oscillatory solutions of a kind of first-order neutral Differential Equation, Mathematical Communication, 22, 151-164, (2017).

9. L. Lietc. al, Bounded Nonoscillatory Solutions for First Order Neutral Delay Differential Equations, Int. Journal of Math. Analysis, 6, 1291 - 1299, (2012).

10. Mohamad H. A. and I. Z. Mushtt, Oscillation of Second Order Nonlinear Neutral Differential Equations, Pure and Applied Mathematics Journal, 4, 62-65 (2015).

of Science and Technology, 34, 22-26, (2018).

11. H. L. Royden and P. M. Fitzpatrick, Real Analysis, Pearson Education Asia Limited and China Machine Press, (2010).

12. S. Tanaka, Existence of Positive Solutions for a Class of First-Order Neutral Functional Differential Equations, Journal of Mathematical Analysis and Applications, 229, 501-518, (1999).

13. Y. Yu, H. Wang, Nonoscillatory Solutions of Second-Order Nonlinear Neutral Delay Equations, J. Math. Anal. Appl., 311, 445-456, (2005).

14. Y. Zhou, B. Zhang, Existence of Nonoscillatory Solutions of Higher-Order Neutral Differential Equations with Positive and Negative Coefficients, Appl. Math. Lett, 15, 867-874, (2002). 
وجود الحل المقيد النسبي الغير متذبذب للمعادلات التفاضلية المحايدة من الرتبة الثاتية

$$
\begin{aligned}
& \text { بشار احمد جواد شربه r } \\
& \text { قسم الرياضيات } \\
& \text { كلية علوم الحاسوب والرياضيات } \\
& \text { جامعة الكوفة وابة }
\end{aligned}
$$

bashara.hamod@uokufa.edu.iq ${ }^{1}$

$$
\begin{aligned}
& \text { حسين علي تحمه ' } \\
& \text { قسم الرياضيات } \\
& \text { كلية العلوم للبنات } \\
& \text { جامعة بغداد }
\end{aligned}
$$

hussainmohamad22@gmail.com2

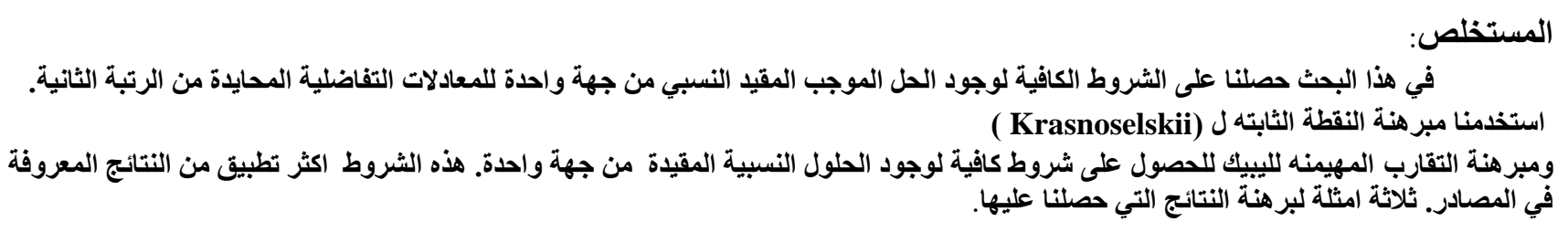

